\title{
Programmed hypertension in rats treated with a NF-кB inhibitor during nephrogenesis: renal mechanisms
}

\author{
Daniele Canale ${ }^{1}$, Mariliza V Rodrigues ${ }^{2}$, Daniele N Ferreira ${ }^{1}$, Flavia G Machado ${ }^{1}$, Mariana M Veras ${ }^{3}$, \\ Denise MAC Malheiros ${ }^{1}$, José E Krieger ${ }^{2}$, Clarice K Fujihara ${ }^{1}$, Gabriela Venturini ${ }^{2}$ and Roberto Zatz ${ }^{1}$
}

Suppression of the renin-angiotensin system (RAS) during murine lactation causes progressive renal injury, indicating a physiological action of angiotensin II on nephrogenesis. The nuclear factor NF-кB system is one of the main intracellular mediators of angiotensin II. We investigated whether inhibition of this system with pyrrolidine dithiocarbamate (PDTC) during rat nephrogenesis would lead to similar hypertension and renal injury as observed with RAS suppressors. Immediately after delivery, 32 Munich-Wistar dams, each nursing 6 male pups, were divided into 2 groups: C, untreated, and PDTC, receiving PDTC, $280 \mathrm{mg} \mathrm{kg}^{-1}$ day $^{-1}$ orally, during 21 days. After weaning, the offspring were followed until 10 months of age without treatment. Adult rats that received neonatal PDTC exhibited stable hypertension and myocardial injury, without albuminuria. To gain additional insight into this process, the renal expression of RAS components and sodium transporters were determined by quantitative real-time PCR (qRT-PCR) at 3 and 10 months of life. Renal renin and angiotensinogen were upregulated at 3 and downregulated at 10 months of age, suggesting a role for early local RAS activation. Likewise, there was early upregulation of the proximal sodium/glucose and sodium/bicarbonate transporters, which abated later in life, suggesting that additional factors sustained hypertension in the long run. The conclusions drawn from the findings were as follows: (1) an intact NF-kB system during nephrogenesis may be essential to normal renal and cardiovascular function in adult life; (2) neonatal PDTC represents a new model of hypertension, lacking overt structural injury or functional impairment of the kidneys; and (3) hypertension in this model seems associated with early temporary activation of renal RAS and sodium transporters.

Hypertension Research (2011) 34, 693-700; doi:10.1038/hr.2011.4; published online 17 February 2011

Keywords: experimental models; kidney; nephrogenesis; neonatal; NF-kB

\section{INTRODUCTION}

Several events occurring during organogenesis can lead to the development of arterial hypertension during adult life. Rodents, in which nephrogenesis is completed during the first 2 weeks after birth, can be programmed during this period to develop hypertension in adult life by maneuvers such as protein restriction ${ }^{1}$ and dietary salt overload. ${ }^{2}$ The mechanisms responsible for blood pressure heightening in this setting are presently obscure, and may involve subtle changes in renal handling of sodium. ${ }^{3}$

Treatment with suppressors of the renin-angiotensin system (RAS) during rat lactation (first 3 weeks after birth) has also been shown to promote hypertension later in life. However, the study of the pathogenesis of blood pressure elevation in these rats is complicated by the concomitant development of severe and progressive glomerular and renal interstitial injury. ${ }^{4-7}$

Several of the inflammatory effects of angiotensin II in adult life are mediated, at the intracellular level, by the nuclear factor NF- $\kappa B$ system. ${ }^{8-11}$ Accordingly, continuous inhibition of this system exerts a protective effect in an experimental model of chronic kidney disease. ${ }^{12}$ However, it is presently unknown whether the NF- $\kappa \mathrm{B}$ system participates in organogenesis and, particularly, whether blockade of this system during nephrogenesis would lead to changes similar to those observed with administration of RAS suppressors.

Given the previously demonstrated effect of RAS suppressors on nephrogenesis, and the fact that the NF- $\mathrm{KB}$ is a well-known intracellular mediator of angiotensin II, we sought to investigate whether inhibition of the NF- $\mathrm{BB}$ system during rat nephrogenesis would lead to similar hypertension and renal injury as observed with suppressors of the RAS.

\section{METHODS}

All experimental procedures were approved by the local research ethics committee (CAPPesq, process no. 669/06) and developed in strict conformity with local institutional guidelines and with well-established international standards for manipulation and care of laboratory animals.

${ }^{1}$ Renal Division, Department of Clinical Medicine, Faculty of Medicine, University of São Paulo, São Paulo, Brazil; ${ }^{2}$ Heart Institute (INCOR), University of São Paulo, São Paulo, Brazil and ${ }^{3}$ Department of Pathology, Faculty of Medicine, University of São Paulo, São Paulo, Brazil

Correspondence: Dr R Zatz, Renal Division, Department of Clinical Medicine, Faculty of Medicine, University of São Paulo, Avenida Dr Arnaldo 455, 3-s/3342, CEP 01246-903, São Paulo, Brazil.

E-mail:rzatz@usp.br

Received 26 April 2010; revised 15 December 2010; accepted 20 December 2010; published online 17 February 2011 


\section{Experimental groups}

A total of 25 adult female Munich-Wistar rats, 10-12 weeks of age and weighing 160-200g, obtained from a local facility at the Faculty of Medicine, University of São Paulo, were housed in individual plastic boxes after mating, receiving unrestrained regular rodent chow containing $0.5 \% \mathrm{Na}$ and $22 \%$ protein (Nuvital Labs, Curitiba, Brazil) and tap water. Temperature was kept at $23 \pm 1{ }^{\circ} \mathrm{C}$ and relative air humidity at $60 \pm 5 \%$, and a $12 / 12 \mathrm{~h} \mathrm{light/dark} \mathrm{cycle}$ was maintained by an electronic timer. After birth, six pups were kept with each dam until weaning, which was 25 days later. During the first 20 days of lactation, the dams received the NF- $\mathrm{KB}$ inhibitor pyrrolidine dithiocarbamate (PDTC), dissolved in the drinking water at $280 \mathrm{mg} \mathrm{kg}^{-1} \mathrm{day}^{-1}$. PDTC had been used in a previous study of adult rats, and found to be effective by the oral route. ${ }^{12}$ In preliminary experiments with lactating rats, PDTC was also shown to be effective by this route, and the dose of $280 \mathrm{mg} \mathrm{kg}^{-1} \mathrm{day}^{-1}$ was found to be the highest dose not causing death or severe growth retardation of dams or pups. After weaning, male rats were kept in cages in groups of no more than four, receiving ad libitum food and water. A total of 34 male offspring from PDTC-treated dams (group PDTC) and 32 age-matched control rats never exposed to PDTC (C) were utilized in these studies.

\section{Main experimental protocol}

A total of $13 \mathrm{C}$ and 12 PDTC rats were studied at 3 months of age for determination of the circulating levels of creatinine $\left(\mathrm{S}_{\mathrm{Cr}}\right)$, sodium $\left(\mathrm{P}_{\mathrm{Na}}\right)$, potassium $\left(\mathrm{P}_{\mathrm{K}}\right)$, plasma renin activity (PRA) and aldosterone (Aldo), urinary sodium $\left(\mathrm{U}_{\mathrm{Na}} \mathrm{V}\right)$ and potassium $\left(\mathrm{U}_{\mathrm{K}} \mathrm{V}\right)$ excretion rates, and histomorphometric analysis of renal and heart tissue. In a second cohort of rats $(13 \mathrm{C}$ and 16 PDTC), all these measurements were repeated at 10 months of age, with assessment of tail-cuff pressure (TCP) and albuminuria $\left(\mathrm{U}_{\mathrm{alb}} \mathrm{V}\right)$ having been performed at 3, 6 and 10 months of age. Rats of neither the control nor the PDTC group received any kind of treatment during these studies, except the PDTC treatment, during lactation only, in group PDTC.

\section{Glomerular counting}

By direct counting. At 3 months of age, rats from a separate cohort (six from group C and six from group PDTC) were anesthetized with ketamine (50 $\mathrm{mg} \mathrm{kg}^{-1}$ intramuscular) and xylazine $\left(10 \mathrm{mg} \mathrm{kg}^{-1}\right.$ intramuscular), and the kidneys were rapidly perfused retrogradely through the abdominal aorta with a $20 \%$ India ink solution as described previously. ${ }^{13}$ A suspension of glomeruli was obtained by immersing the right kidney in an acidic medium and then resuspending the material in saline. ${ }^{13}$ Glomeruli were independently counted under a stereomicroscope at $\times 50$ magnification by two observers (DC and FGM), who were blinded to the experimental groups. The glomerular count attributed to each rat was the average of the two countings thus obtained. The discrepancy between the two countings never exceeded $10 \%$.

By stereology. We estimated the total number of glomeruli per kidney at 3 months of age in 6 rats from each group in serial 4 - $\mu \mathrm{m}$-thick slices of paraffinembedded renal tissue, using an adaptation ${ }^{14}$ of the stereological method described by Sterio. ${ }^{15}$

\section{Analytical techniques}

$\mathrm{U}_{\mathrm{alb}} \mathrm{V}$ was determined by radial immunodiffusion. ${ }^{16}$ At 3 or 10 months of age, rats were anesthetized with ketamine $\left(50 \mathrm{mg} \mathrm{kg}^{-1}\right)$ and xylazine $\left(10 \mathrm{mg} \mathrm{kg}^{-1}\right)$, and blood samples were collected from the abdominal aorta for $\mathrm{S}_{\mathrm{Cr}}$, Aldo, $\mathrm{P}_{\mathrm{Na}}$ and $\mathrm{P}_{\mathrm{K}} . \mathrm{S}_{\mathrm{Cr}}$ was measured by a colorimetric system using a commercial kit (Labtest Diagnostica, São Paulo, Brazil); Aldo was assessed by radioimmunoassay using a commercial kit (Coat-Account Aldosterone; Diagnostic Products, Los Angeles, CA, USA); $\mathrm{P}_{\mathrm{Na}}, \mathrm{P}_{\mathrm{K}}, \mathrm{U}_{\mathrm{Na}} \mathrm{V}$ and $\mathrm{U}_{\mathrm{K}} \mathrm{V}$ were determined with specific electrodes (Easy Lyte Na/K Analyser; Medica, Bedford, MA, USA).

\section{Histomorphometric and immunohistochemistry analysis}

For histomorphometric analysis at 3 or 10 months of age, rats were anesthetized as described previously and the right kidney was removed, immediately frozen in liquid nitrogen and stored at $-70^{\circ} \mathrm{C}$, whereas the left kidney was retrogradely perfusion-fixed from the abdominal aorta. Further details of these procedures are given elsewhere. ${ }^{6}$ Histomorphometric measurements for assessment of glomerular injury and interstitial expansion were performed by a single observer (DMACM) who was blinded to the groups. For each rat, the percentage of glomeruli exhibiting sclerotic lesions was estimated in sections stained by the periodic acid-Schiff reaction. The fraction of the renal cortical area occupied by interstitial tissue was estimated in Masson-stained sections by a point-counting technique. ${ }^{17}$ The total diameter and wall thickness of periglomerular arterioles was measured by a single observer blinded to the groups under $\times 200$ magnification, with the aid of a micrometric scale $(2.5 \mu \mathrm{m}$ smallest division). Immunohistochemical analysis was performed utilizing conventional methods, ${ }^{6}$ employing a polyclonal goat anti-angiotensinogen antibody (Santa Cruz Biotechnology, Santa Cruz, CA, USA) to detect the presence of cortical angiotensinogen (AGT) in renal tissue. The percentage of cortical area staining positive for AGT was quantified blindly with the aid of an image processing software (Image Pro Plus, Media Cybernetics, Bethesda, MD, USA). A total of 25 high-power microscopic fields were examined per section in 5 animals from each experimental group.

To evaluate myocardial tissue damage, the hearts were excised after perfusion-fixation of the kidneys and, after removal of the atria and right ventricles, the left ventricles were weighted and fixed in Dubosq-Brazil solution. The ventricular tissue was then cut into slices and stained with periodic acidSchiff and Masson trichrome to estimate the mean myocyte transversal diameter of the left ventricle and the extent of left ventricular fibrosis. The myocyte transversal diameter for each rat was obtained by measuring the diameters of at least 40 fibers under $\times 400$ magnification, always close to the nucleus. Left ventricular fibrosis was assessed with a point-counting technique under $\times 200$ magnification, and the results were presented as a percentage of the total cortical area. All heart measurements were made by a single observer (DNF), who was blinded to the groups. All morphometrical measurements were performed using a microscope connected to a video monitor.

\section{Measurement of TCP}

TCP was assessed using a commercially available (Visitech Systems, Apex, NC, USA), completely automated method, ${ }^{18}$ under light restraining, and after light warming. Munich-Wistar rats are extremely docile, but to avoid any interference of stress, all rats were carefully preconditioned to the procedure so as to accept it calmly at the time of TCP determination. As an additional precaution, TCP values were always taken as the average of at least three consecutive measurements that varied by no more than $5 \mathrm{~mm} \mathrm{Hg}$, that is, after stabilization of blood pressure.

\section{Assessment of PRA}

To determine PRA, previously trained animals were lightly warmed and restrained, remaining calm during the entire procedure. A $500 \mu \mathrm{l}$ blood sample was then obtained by a skilled person using a 26-gauge butterfly needle, and immediately chilled. Plasma was separated and stored at $-20^{\circ} \mathrm{C}$ until processing. PRA was measured using a commercially available radioimmunoassay kit (Gamma Coat Plasma Renin Activity; Diasorin, Stillwater, MN, USA). In previous experiments, this procedure was shown to yield reliable results. ${ }^{19}$

\section{Renal expression of RAS components and tubular transporters}

Quantitative real-time PCR (qRT-PCR) was performed in frozen renal tissue ( $n=6$ for each group) to analyze the expression of genes deemed likely to be related to the development of hypertension in rats that received neonatal PDTC. The following genes were assessed: $A G T$; renin; angiotensin-converting enzyme (ACE); angiotensin II receptor type 1 isoform a (AT1a); angiotensin II receptor type 1 isoform b (AT1b); proximal sodium/glucose transporter isoform 1 (SGLT1; Slc5a1); proximal sodium/hydrogen exchanger isoform 3 (NHE3; Slc9a3); proximal sodium/bicarbonate transporter (NBC; Slc4a4); proximal sodium/phosphate transporter isoform IIa (NaPi; Slc34al); sodium/chloride cotransporter (NCC; Slc12a3), Henle's loop sodium/potassium/chloride cotransporter (NKCC; Slc12a1); collecting duct epithelial sodium channel type $\beta$ (ENaC; Scnn1b) and collecting duct potassium channel (renal outer medullary potassium (ROMK); Kcnj1). The extraction and preparation of total RNA were performed. For cDNA synthesis, $2 \mu \mathrm{g}$ total RNA and a Superscript III kit (Invitrogen Technologies, São Paulo, Brazil) were employed. Real-time PCR was performed using SYBR Green PCR Master Mix (Applied Biosystem, Foster City, CA, USA) on an ABI PRISM 7500 Fast System (Applied Biosystem). Specific 
Table 1 Sequences of primers used for real-time PCR

\begin{tabular}{|c|c|c|}
\hline Primer & Sense $5^{\prime} \rightarrow 3^{\prime}$ & Antisense $3^{\prime} \rightarrow 5^{\prime}$ \\
\hline AGT & TCTACCCTTTTGGGTGCTG & CAAGGAGGATGCTGTTGAGA \\
\hline Renin & ACGTGAGCATCAGCAAGG & GAGATATAGGATGTGCCAGT \\
\hline$A C E$ & CTTTGACGGAAGCATCACC & CAGGAACGTGGAACTTGGA \\
\hline ATla & CACAACCCTCCCAGAAAGTG & AGGGCCATTTTGTTTTTCTG \\
\hline$A T 1 b$ & GGCTTGAAAGAAGCCCAGA & CGAGGTGAATTGCTCTCTGA \\
\hline SGLT1 (S/c5a1) & ССTGCTGGCTATTTTCTGC & GATGATCTTGGGGCAGTTG \\
\hline NHE3 (SIc9a3) & CTGGCACACAACTTCACCA & ATCCTCGTCAGGGGAGAAC \\
\hline$N B C(S / c 4 a 4)$ & CAATGGACATCACGGAACA & GAGGCACGACTTTCACTGG \\
\hline $\mathrm{NaPi}(\mathrm{S} / \mathrm{c} 34 a 1)$ & CTTCAACATCTCGGGCATC & AGGTAGAGGACGGCAAACC \\
\hline $\operatorname{NCC}(\operatorname{Slc} 12 \mathrm{a} 3)$ & GCAGGTGAGGCTGAATGAG & TGGTTACCTCGGATGAGGA \\
\hline NKCC (S/c12a1) & GCAGAACTGGAAGCAGTCAA & CAGGAGGGAGGTTCTTGGT \\
\hline$E N a C(S c n n 1 b)$ & CGTGTGGCTGCTCTCTAA & CAGGAGGCCACTAGCTTGA \\
\hline ROMK (Kcnj1) & CTTCGGAACGGAGTGTGTT & GCATCCACATTGCCAAACT \\
\hline
\end{tabular}

Abbreviations: ACE, angiotensin-converting enzyme; AGT, angiotensinogen; AT 1a, angiotensin II receptor type 1 isoform a; AT 1b, angiotensin II receptor type 1 isoform b; $E N a C$, epithelial sodium channel type $\beta$; NBC, sodium/bicarbonate cotransporter; NaPi, sodium/phosphate cotransporter isoform Ila; NCC, sodium/chloride cotransporter; NHE3, sodium/hydrogen exchanger isoform 3; NKCC, sodium/potassium/chloride cotransporter; ROMK, potassium channel; SGLT1, sodium/glucose cotransporter isoform 1.

primer pairs, listed in Table 1, were used. All primers were purchased from Invitrogen Technologies. Relative gene expression values were evaluated with the $2^{-\mathrm{DDCt}}$ method $^{20}$ using cyclophylin as housekeeping gene. Results are presented as fold increase in PDTC-treated rats relative to control at 3 and 10 months.

\section{Statistical analysis}

Statistical differences were assessed by two-way analysis of variance, with treatment and time as intervening factors and with pairwise post test comparisons according to the Bonferroni method. ${ }^{21}$ Student's unpaired $t$-test was used in the qRT-PCR experiments, in immunohistochemistry and in nephron number analysis, which involved only simple comparisons between PDTC and $C$ rats. All tests were performed using GraphPad Prism version 4.00 for Windows (GraphPad Software, San Diego, CA, USA). Results are expressed as means \pm s.e.

\section{RESULTS}

At 3 months of age, food consumption was slightly but significantly diminished in rats that received neonatal PDTC (group PDTC) compared with control rats $\left(23.3 \pm 0.6\right.$ vs. $21.2 \pm 0.7 \mathrm{~g} \mathrm{day}^{-1}$, $P<0.05)$. A similar trend was observed at 10 months of age $\left(24.4 \pm 0.7\right.$ vs. $\left.21.4 \pm 1.0 \mathrm{~g} \mathrm{day}^{-1}, P<0.05\right)$. Urine outputs were also similar between groups $\left(26.5 \pm 4.8\right.$ vs. $28.6 \pm 8.3 \mathrm{ml} \mathrm{day}^{-1}$ at 3 months and $31.4 \pm 1.8$ vs. $31.0 \pm 2.2 \mathrm{ml} \mathrm{day}^{-1}$ at 10 months, $\left.P>0.05\right)$. Renal and systemic functional parameters obtained at 3 and 10 months of age are given in Table 2. Although body weight was comparable in groups C and PDTC at weaning, a numerical difference was observed at 3 months, with PDTC rats growing less than controls; at 10 months of age, this difference became significant, indicating mild growth retardation in group PDTC. PRA was significantly diminished in PDTC when compared with control at 3 months of age $(3.1 \pm 0.5$ vs. $\left.5.5 \pm 0.5 \mathrm{AI} \mathrm{ml}^{-1} \mathrm{~h}^{-1}, P<0.05\right)$, but not at 10 months of age. No differences between groups and no effect of time were observed regarding $\mathrm{P}_{\mathrm{Na}}, \mathrm{P}_{\mathrm{K}}, \mathrm{S}_{\mathrm{Cr}}$ or Aldo, indicating that neonatal PDTC treatment did not interfere with long-term renal function and basic homeostasis in adult life. $\mathrm{Na}$ and $\mathrm{K}$ excretion rates were similar between groups at both 3 and 10 months of age, indicating that balance of these ions was maintained along the study.

The time course of TCP is described in Figure 1a. Along the entire study, TCP was significantly higher in group PDTC when compared with control. At 3 months of age, TCP was $\sim 30 \mathrm{~mm} \mathrm{Hg}$ higher in
Table 2 Renal functional and systemic parameters at 3 and 10 months of age

\begin{tabular}{|c|c|c|c|c|}
\hline & \multicolumn{2}{|c|}{3 months } & \multicolumn{2}{|c|}{10 months } \\
\hline & $C(\mathrm{n}=13)$ & PDTC $(\mathrm{n}=12)$ & $C(\mathrm{n}=13)$ & $\operatorname{PDTC}(\mathrm{n}=16)$ \\
\hline BW, g & $246 \pm 11$ & $226 \pm 5$ & $410 \pm 8^{\dagger}$ & $365 \pm 4^{*, \dagger}$ \\
\hline $\mathrm{P}_{\mathrm{Na}}, \mathrm{mmol}^{-1}$ & $142 \pm 2$ & $147 \pm 2$ & $141 \pm 1$ & $144 \pm 1$ \\
\hline $\mathrm{P}_{\mathrm{K}}, \mathrm{mmol} \mathrm{I}^{-1}$ & $5.0 \pm 0.2$ & $5.2 \pm 0.3$ & $4.9 \pm 0.2$ & $4.6 \pm 0.1$ \\
\hline $\mathrm{U}_{\mathrm{Na}} \mathrm{V}, \mathrm{mEq} \mathrm{I}^{-1}$ & $38 \pm 4$ & $45 \pm 5$ & $39 \pm 4$ & $39 \pm 4$ \\
\hline $\mathrm{U}_{\mathrm{K}} \mathrm{V}, \mathrm{mEq} \mathrm{I}^{-1}$ & $85 \pm 10$ & $112 \pm 15$ & $102 \pm 9$ & $98 \pm 6$ \\
\hline $\mathrm{S}_{\mathrm{Cr}}, \mathrm{mg} \mathrm{dl}^{-1}$ & $0.51 \pm 0.02$ & $0.60 \pm 0.02$ & $0.51 \pm 0.04$ & $0.57 \pm 0.04$ \\
\hline Aldo, $\mathrm{pg} \mathrm{ml}^{-1}$ & $340 \pm 36$ & $378 \pm 53$ & $352 \pm 33$ & $374 \pm 38$ \\
\hline PRA, $\mathrm{Al}^{-1} \mathrm{ml}^{-1} \mathrm{~h}^{-1}$ & $5.5 \pm 0.65$ & $3.1 \pm 0.7^{*}$ & $4.5 \pm 0.5$ & $3.9 \pm 0.5$ \\
\hline
\end{tabular}

Abbreviations: Aldo, plasma aldosterone concentration; BW, body weight; $\mathrm{C}$, control rats PDTC, pyrrolidine dithiocarbamate; $P_{K}$, plasma potassium concentration; $P_{\mathrm{Na}}$, plasma sodium concentration; PRA, plasma renin activity; $\mathrm{S}_{\mathrm{cr}}$, serum creatinine concentration; $\mathrm{U}_{\mathrm{K}} \mathrm{V}$, urinary potassium excretion; $\mathrm{U}_{\mathrm{Na}} \mathrm{V}$, urinary sodium excretion.

Values are means \pm s.e. ${ }^{*} P<0.05$ vs. respective control value; ${ }^{\dagger} P<0.05$ vs. respective 3-month value.
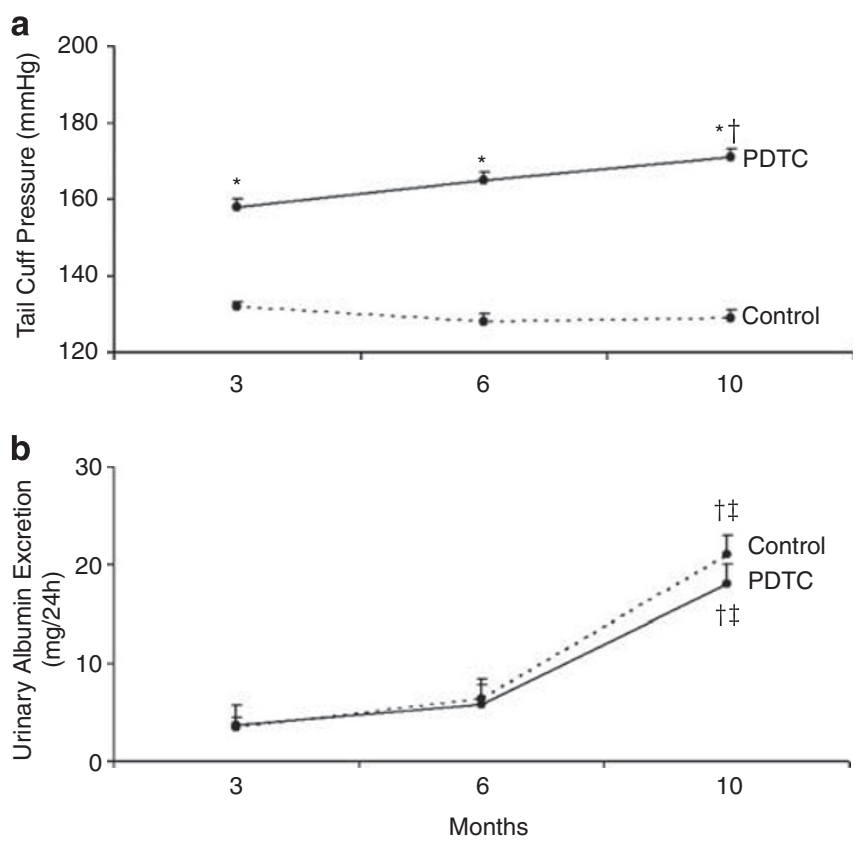

Figure 1 Time course of tail-cuff pressure (TCP; a) and urine albumin excretion rate $\left(U_{\mathrm{alb}} \mathrm{V} ; \mathbf{b}\right)$ in control rats (Control; $\left.n=13\right)$ and in rats treated with pyrrolidine dithiocarbamate (PDTC) during lactation (PDTC; $n=16$ ). ${ }^{*} P<0.05$ vs. respective control value. ${ }^{\dagger} P<0.05$ vs. respective 3 -month value. ${ }^{\ddagger} P<0.05$ vs. respective 6 -month value.

group PDTC than in control, a difference that exceeded $50 \mathrm{~mm} \mathrm{Hg}$ at 10 months of age. Figure $1 \mathrm{~b}$ depicts the time course of urine albumin excretion rate. Albuminuria rose modestly with time in both control and PDTC rats, with no significant difference between groups.

The parameters representing renal structural injury are shown in Figure 2. At both 3 and 10 months of age, rats treated with neonatal PDTC exhibited no increase in the percentage of glomeruli exhibiting sclerotic lesions relative to age-matched controls (Figure 2a). Similarly, no difference was observed between groups regarding interstitial expansion (Figure 2b) or periglomerular arteriolar thickness $(2.6 \pm 0.1 \mu \mathrm{m}$ in control vs. $2.6 \pm 0.1 \mu \mathrm{m}$ in PDTC).

Although absolute values for left ventricle weight were greater in group PDTC than in control (not shown), no difference between 
a
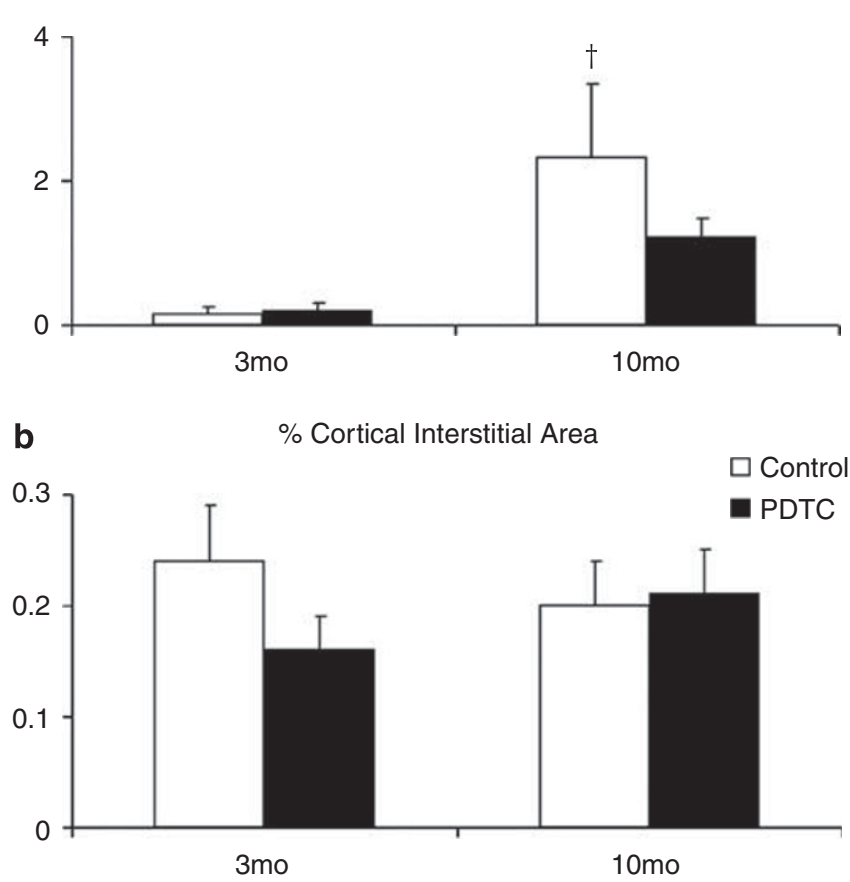

Figure 2 Bar graph representation of the intensity of glomerulosclerosis (a) and renal cortical interstitial expansion (b) in control rats (open bars, $n=13$ at both 3 and 10 months of age) and in rats treated with pyrrolidine dithiocarbamate (PDTC; filled bars, $n=12$ at 3 and $n=16$ at 10 months of age). ${ }^{\dagger} P<0.05$ vs. respective 3 -month value.

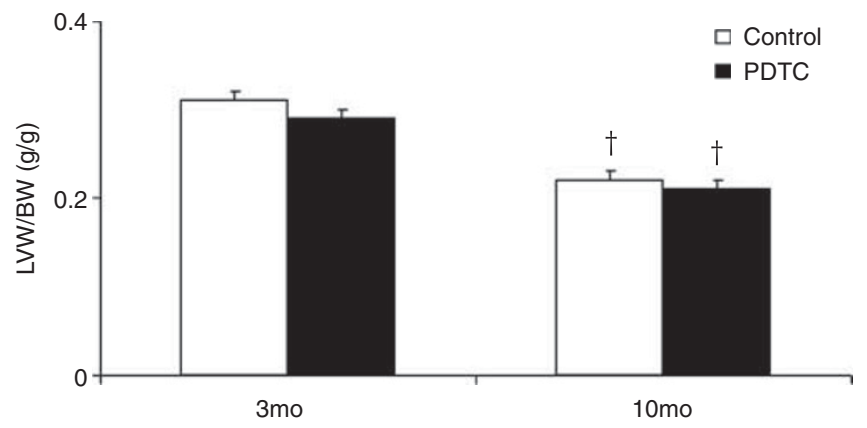

Figure 3 Bar graph representation of the left ventricular weight factored by body weight (LVW/BW) in control rats (open bars, $n=13$ at both 3 and 10 months of age) and in rats treated with pyrrolidine dithiocarbamate (PDTC; filled bars, $n=12$ at 3 and $n=16$ at 10 months of age). ${ }^{\dagger} P<0.05$ vs. respective 3 -month value.

groups was seen at 3 or 10 months of age when individual results were factored for body weight (Figure 3). However, cardiocyte transversal diameter was significantly higher at 10 months of age in group PDTC ( $16.3 \pm 0.5$ vs. $10.7 \pm 0.2 \mu \mathrm{m}$ in $\mathrm{C}, P<0.05$ ), as shown in Figures 4 and 5. Similarly (Figures 6 and 7), the extent of myocardial fibrosis, which was very low and similar between groups at 3 months, was significantly greater in group PDTC at 10 months of age $(1.2 \pm 0.1 \mathrm{vs}$. $0.4 \pm 0.1 \%$ in $\mathrm{C}, P<0.05)$.

\section{Glomerular counting}

The number of nephrons per kidney, obtained at 3 months of age with the India ink infusion technique (Figure 8a) was similar between groups (28 $492 \pm 366$ in PDTC vs. $27347 \pm 237$ in C, $P>0.05$ ). Similar results (26435 \pm 1608 in PDTC vs. $25206 \pm 1535$ in $C, P>0.05$ ) were obtained using a stereological method (Figure $8 \mathrm{~b}$ ), indicating that neonatal PDTC treatment had no effect on the number of nephrons in adult life.

\section{Renal expression of RAS components and tubular transporters}

The renal expression of RAS components and tubular transporters assessed by qRT-PCR is given in Figure 9. AGT and renin were upregulated in group PDTC at 3 months of age, whereas the expression of ACE, AT1a and AT1b was significantly lowered when compared with control (Figure 9a). At 10 months of age, the expression of all RAS components examined was diminished in group PDTC. At 3 months of age, we detected upregulation of two transporters known to be expressed at the proximal tubule: SGLT1, present at the luminal membrane, and the basolateral NBC, expressed at the basolateral membrane (Figure 9b). The expression of $\mathrm{NaPi}$ was slightly but significantly diminished relative to control. No difference between groups was seen at this time regarding the expression of the proximal NHE3, NKCC, present at the thick ascending limb of Henle's loop, NCC, expressed at the distal convoluted tubule, and the luminal ionic channels, ENaC (sodium) and ROMK (potassium), typically observed at principal cells of the connecting tubule and collecting duct. At 10 months of age, the expression of most of the transporters analyzed was significantly reduced in group PDTC relative to control (Figure 9b).

In keeping with the qRT-PCR findings, the percentage of renal cortical AGT assessed by immunohistochemistry was significantly increased in group PDTC at 3 months of age, and reduced in this group at 10 months of age (Figure 10 and online Supplementary Material).

\section{DISCUSSION}

Unlike previous observations with neonatal losartan, ${ }^{6}$ rats treated with PDTC during lactation showed no gross renal structural abnormalities. However, other findings clearly indicate that PDTC did reach the pups through maternal milk. In adult life, these rats developed arterial hypertension, which was present since at least 3 months, and persisted until 10 months, of age. The exact mechanisms underlying the development of hypertension in rats receiving PDTC during lactation are unclear. According to Guyton's theory, ${ }^{22}$ systemic hypertension invariably reflects some limitation to urinary sodium excretion, either absolute or in the face of excess sodium intake. In the present study, food intake was slightly lower than control in rats given neonatal PDTC, suggesting that increased sodium intake in adult life was not a factor in the pathogenesis of hypertension. This is corroborated by the finding that urinary sodium excretion rate was not increased in rats treated with neonatal PDTC. Most importantly, a decrease in sodium excretion is indeed not expected after hypertension becomes stable, even if the pathogenesis of hypertension involves a limitation to sodium excretion, as Guyton's theory predicts that in the steady state, the elevated blood pressure forces sodium excretion to match sodium intake through the pressure natriuresis mechanism, thus restoring balance. ${ }^{23}$

One obvious explanation for the development of hypertension in rats treated with neonatal PDTC could be a reduction in the number of nephrons, such as described previously in human hypertension, ${ }^{23,24}$ in rats treated with neonatal losartan, ${ }^{6,25}$ and in other experimental models of renal disease associated with elevated blood pressure. ${ }^{26,27}$ However, no difference in nephron number between control and hypertensive rats was detected even with the use of a stereological method. Thus, the hypertension observed in rats treated with neonatal 

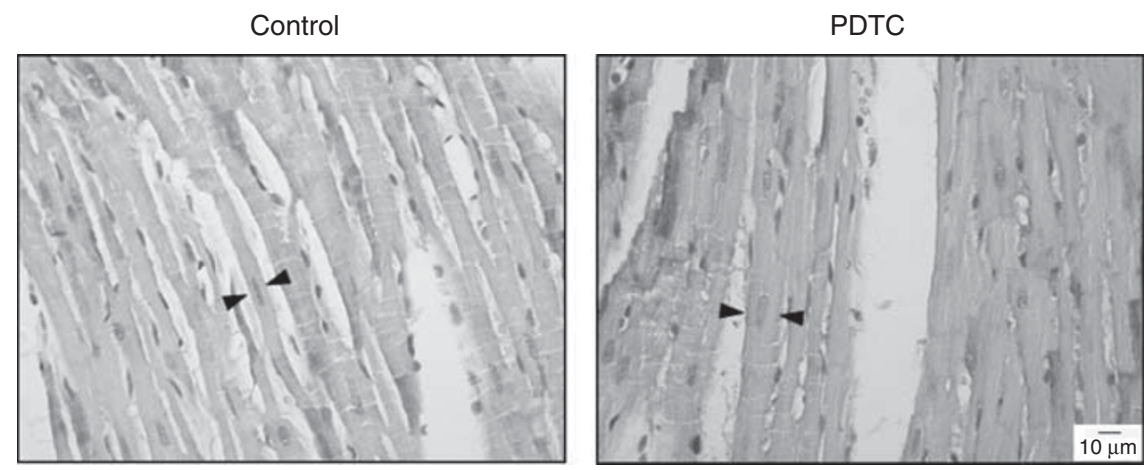

Figure 4 Representative micrographs of the myocardial tissue in control rats and in rats treated with pyrrolidine dithiocarbamate (PDTC) at 10 months of age. The arrowheads delimit representative fiber transversal diameters in each group.

\section{Myocyte Transversal Diameter (Micrometers)}

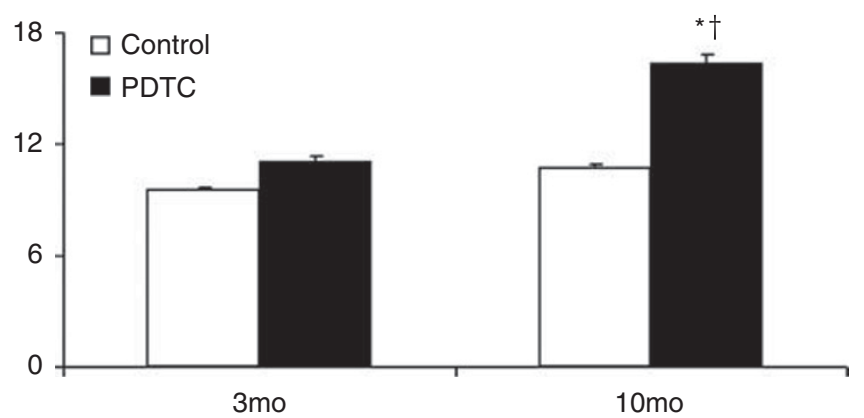

Figure 5 Bar graph representation of the myocyte transversal diameter in control rats (open bars, $n=13$ at both 3 and 10 months of age) and in rats treated with pyrrolidine dithiocarbamate (PDTC; filled bars, $n=12$ at 3 and $n=16$ at 10 months of age). ${ }^{*} P<0.05$ vs. respective control value. $\dagger P<0.05$ vs. respective 3-month value.

PDTC could not be attributed to a reduction in nephron number. It is interesting to note that body weights were modestly decreased in the PDTC group, a finding that was paralleled by a proportional limitation of food intake. Growth stunting has been related to the development of human arterial hypertension. ${ }^{23,24}$ However, this association appears to largely reflect a reduction of the nephron number. Thus, the present findings represent an instance of programmed hypertension with slight limitation of body growth and no reduction of nephron number.

Even without nephron reduction or glomerular injury, a decrease in glomerular filtration rate (GFR) because of glomerular functional changes might have favored sodium retention and blood pressure elevation. Serum creatinine concentrations were similar between control and PDTC rats, both at 3 and 10 months of age, suggesting that GFR was not diminished in rats that received neonatal PDTC. However, as GFRs were not directly measured in this study, the possibility that subtle differences in GFR contributed to the pathogenesis of hypertension in these rats cannot be excluded.

Hypertension in rats treated with neonatal PDTC may have resulted from intensified sodium reabsorption at one or more nephron segments, as shown in experimental models such as the Spontaneously Hypertensive Rat, ${ }^{28-30}$ the Milan Hypertensive Rat ${ }^{31}$ and the Dahl saltsensitive rat. ${ }^{32,33}$ Rats given neonatal PDTC did exhibit evidence of deranged sodium transport in the proximal tubule at 3 months of age. The expression of the $\mathrm{Na}+$ /glucose cotransporter, SGLT1, at the proximal tubule was markedly increased in these animals. Enhanced glucose-dependent sodium transport has been reported in experimental hypertension, ${ }^{33-35}$ and might represent one of the mechanisms involved in blood pressure elevation in this setting. Also consistent with the notion that hypertension in 3-month-old rats may be related to enhanced tubular sodium reabsorption is the observation that these animals showed a significant increase in the expression of the NBC located at the basolateral membrane of the proximal tubule. This observation seems at odds with the finding that the expression of the luminal NHE3 sodium/hydrogen antiporter in this group was similar to control. However, the establishment of an increased rate of sodium (and bicarbonate) transport through the NHE3/NBC axis does not necessarily require upregulation of the NHE3 molecule. The density of the antiporter at the luminal membrane may be increased because of translocation from intracellular organelles, without change in its total abundance in the kidney. ${ }^{36}$ Moreover, the efficiency of the transporter can be enhanced by changes in molecular conformation because of variations in intracellular $\mathrm{pH}^{37}$ or phosphorylation. ${ }^{38}$

An important additional contributing factor to the pathogenesis of hypertension in group PDTC could be the significant increase in the renal expression of renin and AGT at 3 months of age, as augmented local generation of angiotensin II can associate with persistent blood pressure elevation. One of the mechanisms by which angiotensin II might promote hypertension is by enhancing the NHE3 antiporter at the proximal tubule. As pointed out earlier, the finding that the renal NHE3 expression was unchanged in the PDTC group does not necessarily exclude its participation, induced by angiotensin II or not, in the pathogenesis of hypertension in this model: shifting to the apical membrane might enhance proximal sodium transport without increasing the renal abundance of this transporter. It is noteworthy that PRA was decreased in these animals at 3 months of age, suggesting a compensatory response of circulating renin to hypertension and/or to a possible, necessarily slight, expansion of extracellular volume. However, this possibility was not verified in the present study.

The expression of the NaPi cotransporter, also present at the luminal membrane of proximal tubular cells, was significantly decreased at 3 months of age in the PDTC group. At the same time, we detected no change in the expression of Henle's loop's bumetanidesensitive cotransporter, or of the distal tubule's thiazide-sensitive cotransporter. Moreover, the expression of the specific sodium $(\mathrm{ENaC})$ and potassium (ROMK) channels, located at the connecting tubules and collecting ducts, was equally unaltered compared with control. These observations suggest that these transporters are not involved in the pathogenesis of hypertension in the PDTC group, 

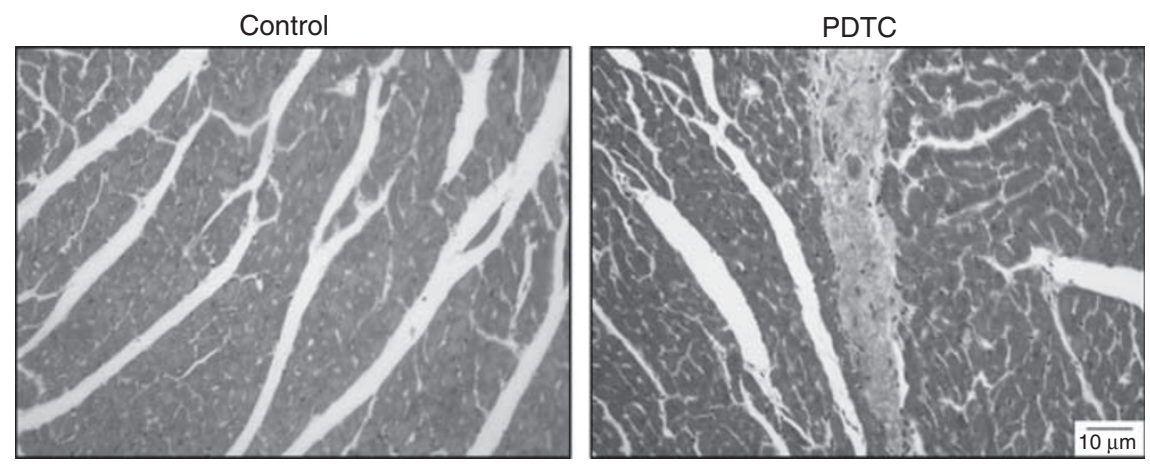

Figure 6 Representative micrographs of left ventricular myocardial tissue in control rats and in rats treated with pyrrolidine dithiocarbamate (PDTC) at 10 months of age, showing a clear streak of fibrosis in the latter.

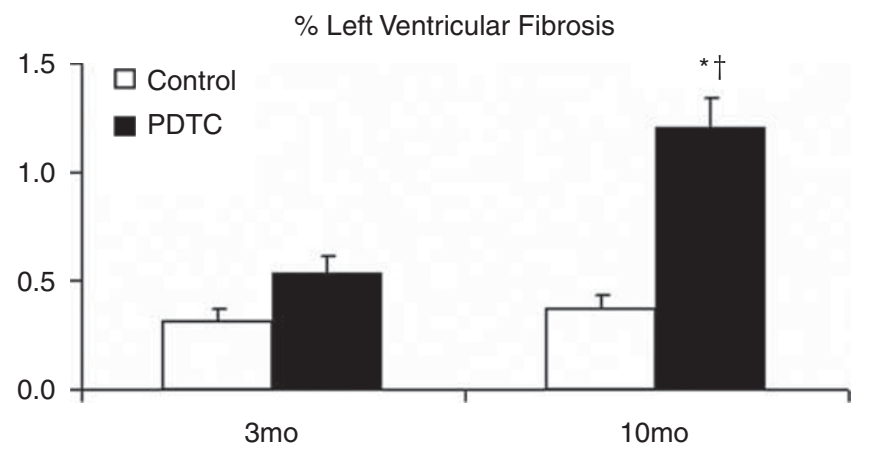

Figure 7 Bar graph representation of the left ventricular myocardial fibrosis in control rats (open bars, $n=13$ at both 3 and 10 months of age) and in rats treated with pyrrolidine dithiocarbamate (PDTC; filled bars, $n=12$ at 3 and $n=16$ at 10 months of age). ${ }^{*} P<0.05$ vs. respective control value. $\uparrow P<0.05$ vs. respective 3-month value.

although changes in internalization and/or affinity of these molecules cannot be ruled out on the basis of the present data.

Upregulation of proximal sodium transporters and RAS activation at the renal tissue were no longer observed at 10 months of age in rats that received neonatal PDTC. At this time, the expression of all the transporters and RAS components studied was markedly decreased compared with control. The reasons for this behavior are unclear. Post-transcriptional abnormalities, leading to dissociation between the expression and the actual availability of these proteins, might explain the apparent reversal of the response observed at 3 months of age. Alternatively, these systems may have undergone an adaptive response later in life, thus limiting sodium transport. However, such a response was apparently unable to reverse blood pressure to normal levels. Therefore, other mechanisms must have contributed to maintain hypertension in the long run in these animals. One plausible explanation is that tubular transporters not examined in this study may have been altered in these animals. An alternative possibility is that elevated blood pressure was perpetuated by renal vascular changes. Although thickening/hyalinization of arteriolar walls and narrowing of arteriolar lumina were not demonstrated in these animals, we cannot exclude the possibility that subtle functional derangement of renal cortical and/or medullary vessels may have promoted slight reduction of renal blood flow and GFR (not detected by measurement of serum creatinine concentration) so as to interfere with sodium excretion and maintain blood pressure at elevated levels. Additional impairment a Number of Nephrons

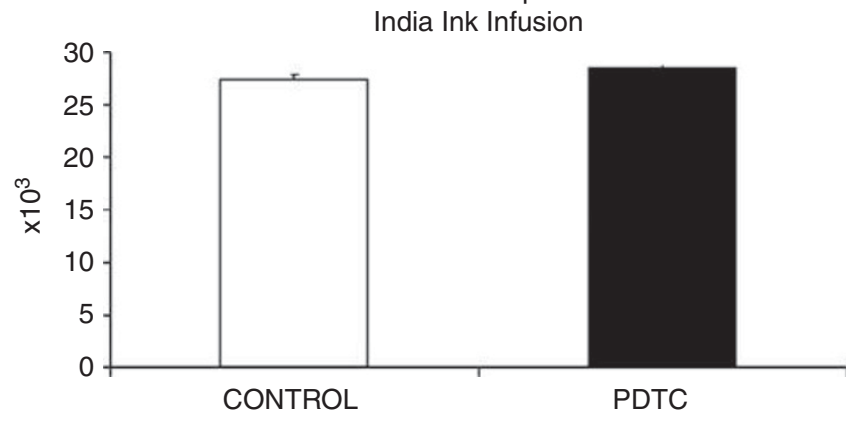

b Number of Nephrons
Stereological Method

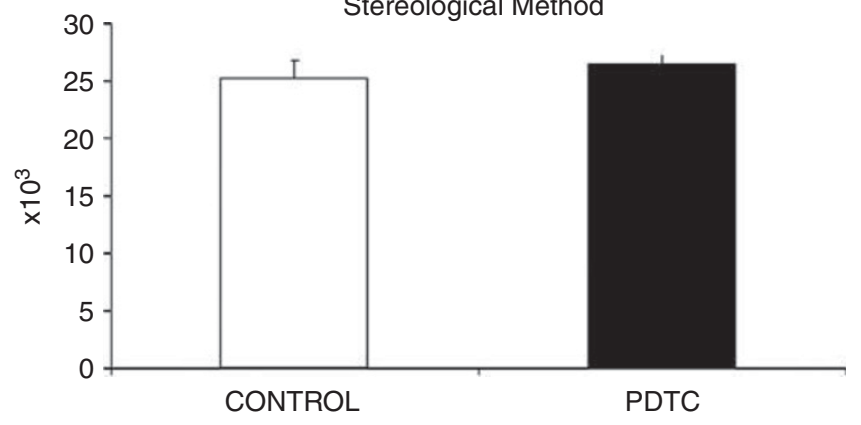

Figure 8 Bar graph representation of the number of nephrons at 3 months of age by India ink infusion (a) and a stereological method (b) in control rats (open bars, $n=6$ for each method) and in rats treated with pyrrolidine dithiocarbamate (PDTC; filled bars, $n=6$ for each method).

of vascular function may have resulted from endothelial damage, reduction of nitric oxide production and/or the presence of increased circulating levels of endogenous nitric oxide synthase inhibitors. ${ }^{39}$ Further studies are necessary to verify these hypotheses.

The moderately elevated blood pressure levels observed in this study were not associated with glomerulosclerosis in excess of that observed in age-matched controls. Although apparently paradoxical, this finding is consistent with previous observations in other models of hypertension. In the Spontaneously Hypertensive Rat model, in which hypertension is much more severe than in the present model, glomerulosclerosis becomes significant only at advanced ages. ${ }^{40}$ Similarly, in the Milan Hypertensive Rat, glomerulosclerosis is absent despite markedly elevated blood pressure levels. ${ }^{41}$ This refractoriness 
a

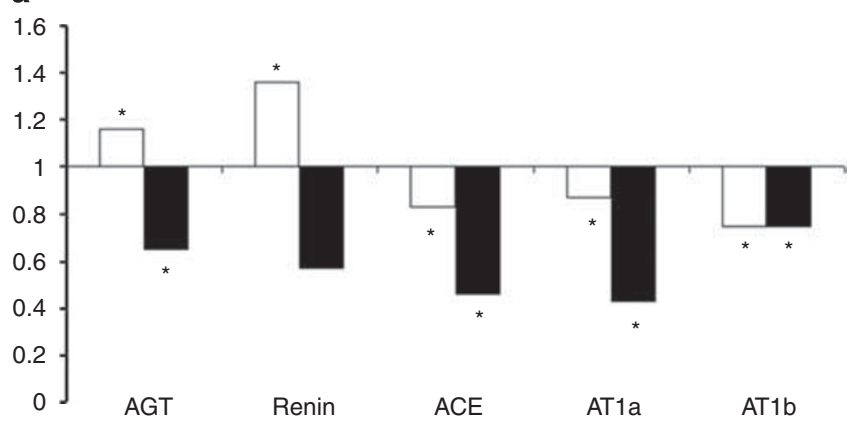

b

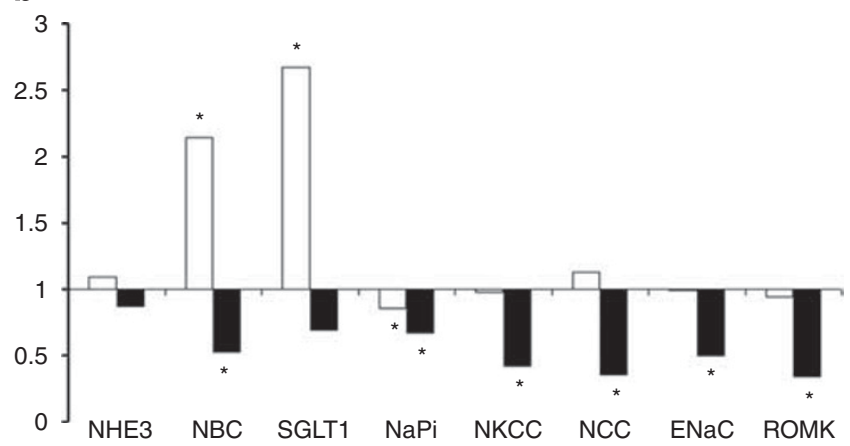

Figure 9 Bar graph representation of the renal mRNA expression of components of (a) renin-angiotensin system (RAS) and (b) tubular transporters in pyrrolidine dithiocarbamate (PDTC)-treated animals at 3 months of age (open bars, $n=6$ ) and PDTC-treated animals at 10 months of age (filled bars, $n=6$ ). Values are represented as fold increase relative to control. ${ }^{*} P<0.05$ vs. respective control.

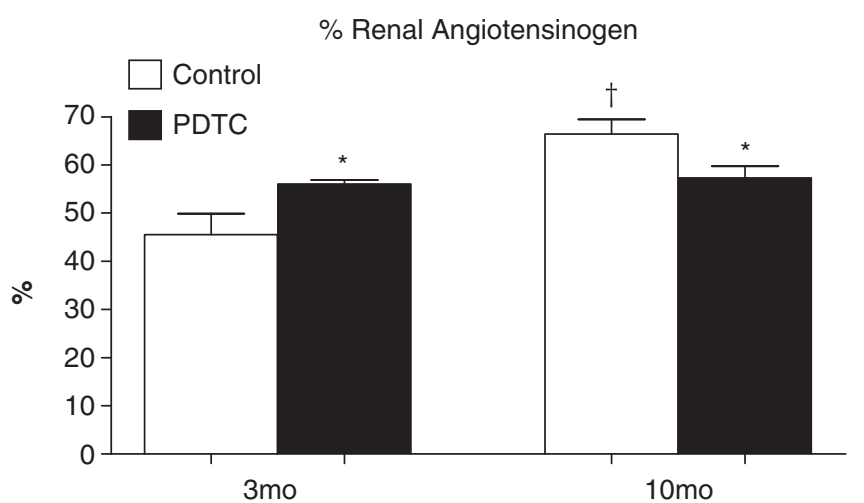

Figure 10 Bar graph representation of the renal expression of angiotensinogen, identified by immunohistochemistry in control animals at 3 months of age (open bars, $n=5$ at both 3 and 10 months of age) and pyrrolidine dithiocarbamate (PDTC)-treated animals (filled bars, $n=5$ at both 3 and 10 months of age). ${ }^{*} P<0.05$ vs. respective control value. ${ }^{\dagger} P<0.05$ vs. respective 3 -month value.

to hypertension-induced glomerular injury is associated with increased renal vascular resistance, reflecting vasoconstriction of the preglomerular vessels and lesser transmission of systemic pressure to the glomerular capillaries, ${ }^{42,43}$ a protective mechanism that may have been operative in the present study as well. In contrast, the Dahl saltsensitive rat, in which this hemodynamic adaptation is defective, develops severe glomerular injury. ${ }^{44}$
As in previous studies of moderate hypertension, ${ }^{45-47}$ gross hypertrophy of the left ventricle was not observed in rats that received neonatal PDTC. This finding also mimics observations made in human hypertensive subjects, a substantial proportion of which may show no clinical signs of left ventricular hypertrophy. ${ }^{48}$ However, microscopic examination of the myocardial tissue revealed a modest but significant increase in cardiocyte transversal diameter at 10 months of age, with a large degree of overlapping between groups, as shown in Figures 4 and 5. Moreover, focal myocardial fibrosis was clearly seen and quantified at this phase. These findings indicate that incipient cardiac hypertrophy and damage to the myocardial tissue were already taking place in these animals, and would be expected to result in overt hypertrophy and severe myocardial fibrosis had the study been conducted for a longer period of time. The fact that these incipient structural changes were seen only at 10 months of age strongly suggests that they resulted simply from long-term exposure to the moderate but persistent elevation of blood pressure, although the possibility that myocardial injury resulted from a direct effect of NF- $\kappa \mathrm{B}$ inhibition during organogenesis ${ }^{49}$ cannot be excluded.

In summary, this study suggests that at least in rats, a functioning $\mathrm{NF}-\kappa \mathrm{B}$ system during nephrogenesis may be essential to a fully normal renal and cardiovascular operation in adult life. Neonatal PDTC treatment may constitute a new model of hypertension, in several respects similar to human essential hypertension. This is a unique model in that it is associated with no gross renal structural injury or functional impairment, nor is it accompanied by a reduction in nephron number. Consistent with Guyton's theory, blood pressure elevation in this model is associated with renal sodium abnormalities such as early local renal activation of RAS and sodium transporters. This model requires no drug administration or surgical procedure, while hypertension is mild and nonprogressive, which favors focusing on high blood pressure itself and on its pathogenesis. For its simplicity and reproducibility, this new model may become a useful tool in the study of the mechanisms of arterial hypertension and in the search for novel therapeutic options.

\section{CONFLICT OF INTEREST}

The authors declare no conflict of interest.

\section{ACKNOWLEDGEMENTS}

We thank Camilla Fanelli for her superb technical assistance. We are also indebted to Claudia Ramos Sena, Simone Ribeiro da Costa and Bianca Helena Ventura Fernandes for their valuable technical help.

This study was supported by Grants 478529/2007-4 and 304657/2007-7 from the Brazilian Council of Scientific and Technologic Development (CNPq).

1 Woods LL, Ingelfinger JR, Nyengaard JR, Rasch R. Maternal protein restriction suppresses the newborn renin-angiotensin system and programs adult hypertension in rats. Pediatr Res 2001; 49: 460-467.

2 Stewart T, Ascani J, Craver RD, Vehaskari VM. Role of postnatal dietary sodium in prenatally programmed hypertension. Pediatr Nephrol 2009; 24: 1727-1733.

3 Manning J, Beutler K, Knepper MA, Vehaskari VM. Upregulation of renal BSC1 and TSC in prenatally programmed hypertension. Am J Physiol Renal Physiol 2002; 283: F202-F206.

4 Chen $Y$, Lasaitiene D, Friberg P. The renin-angiotensin system in kidney development. Acta Physiol Scand 2004; 181: 529-535.

5 Hilgers KF, Norwood VF, Gomez RA. Angiotensin's role in renal development. Semin Nephrol 1997; 17: 492-501.

6 Machado FG, Poppi EP, Fanelli C, Malheiros DM, Zatz R, Fujihara CK. AT1 blockade during lactation as a model of chronic nephropathy: mechanisms of renal injury. Am J Physiol Renal Physiol 2008; 294: F1345-F1353.

7 Matsusaka T, Miyazaki Y, Ichikawa I. The renin angiotensin system and kidney development. Annu Rev Physiol 2002; 64: 551-561. 
8 Gomez-Garre D, Largo R, Tejera N, Fortes J, Manzarbeitia F, Egido J. Activation of NF-kappaB in tubular epithelial cells of rats with intense proteinuria: role of angiotensin II and endothelin-1. Hypertension 2001; 37: 1171-1178.

9 Mezzano S, Aros C, Droguett A, Burgos ME, Ardiles L, Flores C, Schneider H, Ruiz-Ortega M, Egido J. NF-kappaB activation and overexpression of regulated genes in human diabetic nephropathy. Nephrol Dial Transplant 2004; 19: 2505-2512.

10 Ruiz-Ortega M, Bustos C, Hernandez-Presa MA, Lorenzo O, Plaza JJ, Egido J. Angiotensin II participates in mononuclear cell recruitment in experimental immune complex nephritis through nuclear factor-kappa B activation and monocyte chemoattractant protein-1 synthesis. J Immunol 1998; 161: 430-439.

11 Ruiz-Ortega M, Lorenzo O, Ruperez M, Konig S, Wittig B, Egido J. Angiotensin II activates nuclear transcription factor kappaB through AT(1) and AT(2) in vascular smooth muscle cells: molecular mechanisms. Circ Res 2000; 86: 1266-1272.

12 Fujihara CK, Antunes GR, Mattar AL, Malheiros DM, Vieira Jr JM, Zatz R. Chronic inhibition of nuclear factor-kappaB attenuates renal injury in the $5 / 6$ renal ablation model. Am J Physiol Renal Physiol 2007; 292: F92-F99.

13 Gilbert JS, Lang AL, Grant AR, Nijland MJ. Maternal nutrient restriction in sheep: hypertension and decreased nephron number in offspring at 9 months of age. J Physiol 2005; 565: 137-147.

14 Nyengaard JR. Stereologic methods and their application in kidney research. J Am Soc Nephrol 1999; 10: 1100-1123.

15 Sterio DC. The unbiased estimation of number and sizes of arbitrary particles using the disector. J Microsc 1984; 134: 127-136.

16 Mancini G, Carbonara AO, Heremans JF. Immunochemical quantitation of antigens by single radial immunodiffusion. Immunochemistry 1965; 2: 235-254.

17 Jepsen FL, Mortensen PB. Interstitial fibrosis of the renal cortex in minimal change lesion and its correlation with renal function. A quantitative study. Virchows Arch $A$ Pathol Anat Histol 1979; 383: 265-270.

18 Krege JH, Hodgin JB, Hagaman JR, Smithies O. A noninvasive computerized tail-cuff system for measuring blood pressure in mice. Hypertension 1995; 25: 1111-1115.

19 Fujihara CK, Michellazzo SM, de Nucci G, Zatz R. Sodium excess aggravates hypertension and renal parenchymal injury in rats with chronic NO inhibition. Am J Physiol 1994; 266: F697-F705.

20 Livak KJ, Schmittgen TD. Analysis of relative gene expression data using real-time quantitative PCR and the 2(-Delta Delta C(T)) method. Methods 2001; 25: 402-408.

21 Wallenstein S, Zucker CL, Fleiss JL. Some statistical methods useful in circulation research. Circ Res 1980; 47: 1-9.

22 Guyton AC. Kidneys and fluids in pressure regulation. Small volume but large pressure changes. Hypertension 1992; 19: I2-18.

23 Brenner BM, Garcia DL, Anderson S. Glomeruli and blood pressure. Less of one, more the other? Am J Hypertens 1988; 1: 335-347.

24 Keller G, Zimmer G, Mall G, Ritz E, Amann K. Nephron number in patients with primary hypertension. N Engl J Med 2003; 348: 101-108.

25 Tufro-McReddie A, Romano LM, Harris JM, Ferder L, Gomez RA. Angiotensin II regulates nephrogenesis and renal vascular development. Am J Physiol 1995; 269: F110-F115.

26 Dworkin LD, Hostetter TH, Rennke HG, Brenner BM. Hemodynamic basis for glomerular injury in rats with desoxycorticosterone-salt hypertension. J Clin Invest 1984; 73: 1448-1461.

27 Shimamura T, Morrison AB. A progressive glomerulosclerosis occurring in partial five-sixths nephrectomized rats. Am J Pathol 1975; 79: 95-106.

28 Kelly MP, Quinn PA, Davies JE, Ng LL. Activity and expression of $\mathrm{Na}(+)-\mathrm{H}+$ exchanger isoforms 1 and 3 in kidney proximal tubules of hypertensive rats. Circ Res 1997; 80: 853-860.

29 Kim SW, Wang W, Kwon TH, Knepper MA, Frokiaer J, Nielsen S. Increased expression of ENaC subunits and increased apical targeting of AQP2 in the kidneys of spontaneously hypertensive rats. Am J Physiol Renal Physiol 2005; 289: F957-F968.
30 Sonalker PA, Tofovic SP, Jackson EK. Increased expression of the sodium transporter BSC-1 in spontaneously hypertensive rats. J Pharmacol Exp Ther 2004; 311: 1052-1061.

31 Capasso G, Rizzo M, Garavaglia ML, Trepiccione F, Zacchia M, Mugione A, Ferrari P, Paulmichl M, Lang F, Loffing J, Carrel M, Damiano S, Wagner CA, Bianchi G, Meyer G. Upregulation of apical sodium-chloride cotransporter and basolateral chloride channels is responsible for the maintenance of salt-sensitive hypertension. Am J Physiol Renal Physiol 2008; 295: F556-F567.

32 Hoagland KM, Flasch AK, Dahly-Vernon AJ, dos Santos EA, Knepper MA, Roman RJ. Elevated BSC-1 and ROMK expression in Dahl salt-sensitive rat kidneys. Hypertension 2004; 43: 860-865.

33 Ikari A, Tachihara Y, Kawano K, Suketa Y. Differential regulation of $\mathrm{Na}(+), \mathrm{K}(+)$-ATPase and the $\mathrm{Na}(+)$-coupled glucose transporter in hypertensive rat kidney. Biochim Biophys Acta 2001; 1510: 118-124.

34 Bakris GL, Fonseca VA, Sharma K, Wright EM. Renal sodium-glucose transport: role in diabetes mellitus and potential clinical implications. Kidney Int 2009; 75 : 1272-1277.

35 Bautista R, Manning R, Martinez F, Avila-Casado Mdel C, Soto V, Medina A, Escalante B. Angiotensin II-dependent increased expression of $\mathrm{Na}$-glucose cotransporter in hypertension. Am J Physiol Renal Physiol 2004; 286: F127-F133.

36 Donowitz M, Li X. Regulatory binding partners and complexes of NHE3. Physiol Rev 2007; 87: 825-872.

37 Alexander RT, Malevanets A, Durkan AM, Kocinsky HS, Aronson PS, Orlowski J, Grinstein S. Membrane curvature alters the activation kinetics of the epithelial $\mathrm{Na}+$ / $\mathrm{H}+$ exchanger, NHE3. J Biol Chem 2007; 282: 7376-7384.

38 Yang LE, Sandberg MB, Can AD, Pihakaski-Maunsbach K, McDonough AA. Effects of dietary salt on renal $\mathrm{Na}+$ transporter subcellular distribution, abundance, and phosphorylation status. Am J Physiol Renal Physiol 2008; 295: F1003-F1016.

39 Veresh Z, Racz A, Lotz G, Koller A. ADMA impairs nitric oxide-mediated arteriolar function due to increased superoxide production by angiotensin II-NAD(P)H oxidase pathway. Hypertension 2008; 52: 960-966.

40 Feld LG, Cachero S, Van Liew JB, Zamlauski-Tucker M, Noble B. Enalapril and renal injury in spontaneously hypertensive rats. Hypertension 1990; 16: 544-554.

41 Pugliese G, Ricci C, lacobini C, Menini S, Fioretto P, Ferrandi M, Giardino LA Armelloni S, Mattinzoli D, Rastaldi MP, Pugliese F. Glomerular barrier dysfunction in glomerulosclerosis- resistant Milan rats with experimental diabetes: the role of renal haemodynamics. J Pathol 2007; 213: 210-218.

42 Arendshorst WJ, Beierwaltes WH. Renal and nephron hemodynamics in spontaneously hypertensive rats. Am J Physiol 1979; 236: F246-F251.

43 Pugliese G, Pricci F, Barsotti P, lacobini C, Ricci C, Oddi G, Romeo G, Leto G, Marano G, Sorcini M, Sabbatini M, Fuiano G, Di Mario U, Pugliese F. Development of diabetic nephropathy in the Milan normotensive strain, but not in the Milan hypertensive strain: possible permissive role of hemodynamics. Kidney Int 2005; 67: 1440-1452.

44 Takenaka T, Forster H, De Micheli A, Epstein M. Impaired myogenic responsiveness of renal microvessels in Dahl salt-sensitive rats. Circ Res 1992; 71: 471-480.

45 Cleary MP. Consequences of restricted feeding/refeeding cycles in lean and obese female Zucker rats. J Nutr 1986; 116: 290-303.

46 Reddy SR, Kotchen TA. Dietary sodium chloride increases blood pressure in obese Zucker rats. Hypertension 1992; 20: 389-393.

47 Arnal JF, el Amrani Al, Chatellier G, Menard J, Michel JB. Cardiac weight in hypertension induced by nitric oxide synthase blockade. Hypertension 1993; 22: 380-387.

48 Weber MA, Drayer JI, Baird WM. Echocardiographic evaluation of left ventricular hypertrophy. J Cardiovasc Pharmacol 1986; 8: S61-S68.

49 Hernandez-Gutierrez S, Garcia-Pelaez I, Zentella-Dehesa A, Ramos-Kuri M, HernandezFranco P, Hernandez-Sanchez F, Rojas E. NF-kappaB signaling blockade by Bay 11-7085 during early cardiac morphogenesis induces alterations of the outflow tract in chicken heart. Apoptosis 2006; 11: 1101-1109.

Supplementary Information accompanies the paper on Hypertension Research website (http://www.nature.com/hr) 